\title{
Prevalência e fatores associados ao sobrepeso e obesidade em adolescentes a partir de inquéritos populacionais de base escolar
}

\author{
Carolina Cunha Prado \\ Discente da Faculdade de Medicina da Pontifícia Universidade Católica de Campinas (PUC-Campinas) \\ Bolsista PIBIC/CNPq (2018-2019) \\ Carla Cristina Enes \\ Docente permanente do Programa de Pós-graduação em Ciências da Saúde da Pontifícia Universidade Católica \\ de Campinas (PUC-Campinas) \\ Luciana Bertoldi Nucci \\ Docente permanente do Programa de Pós-graduação em Ciências da Saúde da Pontifícia Universidade Católica \\ de Campinas (PUC-Campinas) \\ $\triangle$ luciananucci@puc-campinas.edu.br
}

Recebido em 11 de novembro de 2020

Aceito em 6 de julho de 2021

\begin{abstract}
Resumo:
De acordo com a Organização Mundial da Saúde (OMS), 18\% das crianças e adolescentes de 5-19 anos apresentavam condições de sobrepeso e obesidade em todo o mundo em 2016. Uma importante ferramenta de acompanhamento das condições de saúde de uma população são os inquéritos nacionais de base escolar, que já são realizados para o acompanhamento da prevalência do excesso de peso em adolescentes em vários países. Portanto, o objetivo desse estudo é descrever a prevalência de excesso de peso em crianças e adolescentes a partir de inquéritos nacionais de base escolar. Trata-se de uma revisão sistemática de estudos transversais com amostra de escolares com representatividade nacional. A busca foi realizada a partir das bases eletrônicas PUBMED/MEDLINE, LILACS e SciELO e incluiu artigos publicados de Jan/2014 a Mai/2019 com dados de prevalência de sobrepeso e obesidade. Foram identificadas 204 referências e incluídos na revisão 29 artigos que analisaram dados de 11 inquéritos realizados em países de quatro continentes (América, Ásia, Europa e África). A prevalência de excesso de peso (sobrepeso + obesidade) em crianças e adolescentes variou de $2 \%$ a $37 \%$ nos estudos avaliados. A comparação dos resultados de inquéritos populacionais de base escolar com abrangência nacional é uma valiosa fonte de informação para o monitoramento das condições de saúde de crianças e adolescentes, especialmente se mantidas a temporalidade de realização e a comparabilidade desses inquéritos com dados de outros países.

Palavras-chave: Saúde do Adolescente, Revisão Sistemática, Inquéritos Epidemiológicos, Obesidade, Sobrepeso.

\section{Prevalence of overweight and obesity in adolescents and factors associated with these conditions from school-based population surveys}

\begin{abstract}
:
According to the World Health Organization (WHO), 18\% of children worldwide aged 5-19 years were overweight and obese in 2016. National school-based surveys are an important tool for monitoring the health conditions of a population and they are already being conducted to monitor the prevalence of overweight in adolescents in several countries. The objective of this study is to describe the prevalence of overweight and obesity in children and adolescents reported from national schoolbased surveys. Systematic review of cross-sectional studies with a nationally representative sample of schoolchildren. The search was performed from the electronic databases MEDLINE/PUBMED,
\end{abstract}


LILACS and SciELO and included articles published from January/2014 to May/2019 that reported data of overweight and obesity prevalence. Two hundred and four references were identified and 29 papers that described data of 11 school-based national surveys applied in countries of four continents (America, Asia, Europe, and Africa) were included in the review. The prevalence of overweight and obesity in children and adolescents ranged from $2 \%$ to $37 \%$ in the evaluated studies. Conducting school-based national surveys is a valuable source of information for monitoring the health conditions of children and adolescents, especially if the timelines and comparability of these surveys with data from other countries are maintained.

Keywords: Adolescent Health, Systematic Review, Health Surveys, Obesity, Overweight.

\section{Prevalencia y factores asociados con sobrepeso y obesidad en adolescentes en encuestas de población escolar}

\section{Resumen:}

Según la Organización Mundial de la Salud (OMS), el 18\% de los niños de 5 a 19 años tenían sobrepeso y obesidad en todo el mundo en 2016. Una herramienta importante para controlar las condiciones de salud de esta población, para tanto se están realizando encuestas nacionales en las escuelas para vigilar la prevalencia de sobrepeso en adolescentes en varios países. El propósito de este estudio es describir la prevalencia del sobrepeso en niños y adolescentes a partir de encuestas escolares nacionales. Revisión sistemática de estudios transversales con muestras de escolares representativa a nivel nacional. La búsqueda se realizó en bases de datos electrónicas MEDLINE, LILACS y SciELO e incluyó artículos publicados desde enero de 2014 hasta mayo de 2019 con datos sobre la prevalencia de sobrepeso. Se encontraron 204 referencias y se incluyeron 29 artículos con 11 encuestas en países de cuatro continentes (América, Asia, Europa y África). La prevalencia de sobrepeso en niños y adolescentes varió del $2 \%$ al $37 \%$ en los estudios analizados. La realización de encuestas nacionales de población en las escuelas es una fuente valiosa de información para vigilar las condiciones de salud de los niños y adolescentes, especialmente si se mantiene la temporalidad y la comparabilidad de estas encuestas con los datos de otros países.

Palabras clave: Salud del Adolescente, Revisión Sistemática, Encuestas Epidemiológicas, Obesidad, Sobrepeso.

\section{INTRODUÇÃo}

De acordo com a Organização Mundial da Saúde (OMS), a prevalência mundial de sobrepeso e obesidade em adultos maiores de 18 anos era de $39 \%$ e 13\%, respectivamente, em 2016, atingindo cerca de 1.9 bilhões de adultos (WORLD HEALTH ORGANIZATION, 2018). Entre crianças e adolescentes, a prevalência de obesidade aumentou dez vezes no período de 1975 e 2016 em nível mundial. Em 2016, mais de 340 milhões de jovens com idade entre 5 e 19 anos apresentavam sobrepeso ou obesidade (NCD RISK FACTOR COLLABORATION (NCD-RISC), 2017).

Embora a condição de sobrepeso e obesidade seja um problema de saúde pública que afeta diversas faixas etárias, é entre crianças e adolescentes que ela desempenha um papel mais importante, devido à complexidade do tratamento, à alta probabilidade de persistência 
na vida adulta e à associação com outras doenças crônicas com início precoce como diabetes tipo 2, doenças cardiovasculares, problemas ortopédicos, dentre outros (DANIELS; HASSINK, 2015; SOCIEDADE BRASILEIRA DE PEDIATRIA; DEPARTAMENTO CIENTÍFICO DE NUTROLOGIA, 2012).

O monitoramento da prevalência dessa condição na população ocorre tanto em países desenvolvidos quanto naqueles em desenvolvimento por várias razões: 1- a identificação de níveis elevados de obesidade em muitos países já foi detectada há mais de 20 anos; 2- trata-se de uma condição que pode ser prevenida e controlada a partir de mudanças comportamentais; e 3- os riscos elevados do desenvolvimento de doenças associadas à obesidade, como diabetes tipo 2, hipertensão, doenças coronarianas, muitas formas de câncer e disfunções cognitivas (MITCHELL et al., 2011).

Uma importante ferramenta de acompanhamento das condições de saúde de uma população, incluindo o estado nutricional, são os inquéritos populacionais, que se caracterizam como estudos de base populacional com delineamento transversal, plano de amostragem em múltiplos estágios, e que são realizados quando as informações existentes são inadequadas ou insuficientes para se entender e/ou monitorar uma situação de saúde (FUNDAÇÃO NACIONAL DE SAÚDE, 2002; HULLEY et al., 2015; SZWARCWALD; DAMACENA, 2008).

A frequência com que os inquéritos populacionais são realizados tem aumentado e vários países já o fazem regularmente, permitindo assim o monitoramento de várias condições de saúde e seus fatores de risco (BRASIL et al., 2018; CENTERS FOR DISEASE CONTROL AND PREVENTION (CDC), 2018, 2019; IANNOTTI, 2013; IBGE; COORDENAÇÃO DE POPULAÇÃO E INDICADORES SOCIAIS, 2016; KIM et al., 2016; WORLD HEALTH ORGANIZATION, 2013a). Além disso, a metodologia empregada em estudos dessa natureza permite a otimização de recursos, sem perder a representatividade nacional, a exemplo dos inquéritos telefônicos (BRASIL et al., 2018; CENTERS FOR DISEASE CONTROL AND PREVENTION (CDC), 2018) e inquéritos de base escolar (CENTERS FOR DISEASE CONTROL AND PREVENTION (CDC), 2019; IBGE; COORDENAÇÃO DE POPULAÇÃO E INDICADORES SOCIAIS, 2016; KIM et al., 2016).

Considerando que o acompanhamento da prevalência de sobrepeso e obesidade em crianças e adolescentes já é realizado em vários países a partir de inquéritos populacionais de base escolar, a identificação de inquéritos de abrangência nacional que sejam 
metodologicamente comparáveis torna-se uma valiosa fonte de informação de monitoramento global e a comparação dos resultados desses inquéritos permite não apenas a identificação dos fatores comuns, mas a identificação de fatores que podem estar relacionados aos hábitos culturais de cada país.

Diante desse contexto, o objetivo desta revisão foi identificar estudos que analisaram dados de inquéritos populacionais de base escolar com representatividade nacional, que reportassem a prevalência de sobrepeso e obesidade em crianças e adolescentes e avaliar os fatores de risco e proteção associados à essa condição.

\section{MATERIAL E MÉTODOS}

Foi realizada uma revisão sistemática de artigos científicos, com a participação de dois revisores trabalhando de forma independente. Esta revisão seguiu os critérios do Preferred Reporting Items for Systematic Reviews and Meta-Analyses Check-list (PRISMA) (GALVÃO et al., 2015).

\section{Critérios de elegibilidade}

Foram considerados elegíveis os artigos que atendiam aos seguintes critérios de inclusão: 1) estudos observacionais; 2) utilizaram dados de inquéritos populacionais de base escolar com representatividade nacional do país em que foi aplicado; 3) estimaram a prevalência de excesso de peso (sobrepeso e/ou obesidade) e identificaram os fatores associados; 4) amostra de escolares que frequentavam o equivalente ao $6^{\circ}$ ano do Ensino Fundamental até a terceira série do Ensino Médio (faixa etária aproximada de 13 a 17 anos); 5) publicados nos últimos 5 anos.

Os critérios considerados para a classificação das categorias de IMC dos adolescentes foram os valores específicos para idade e sexo propostos pela Organização Mundial da Saúde (OMS)(WORLD HEALTH ORGANIZATION, 2013b), pelo International Obesity Task Force (IOTF)(COLE; LOBSTEIN, 2012), pelo Working Group on Obesity in China (WGOC)(JI, 2008), pelo Centers for Disease Control and Prevention (CDC)(KUCZMARSKI et al., 2002) ou por Cole et al. 
(2000)(COLE, 2000). Excesso de peso foi definido como a soma das categorias de sobrepeso e obesidade e, quando disponível, essas prevalências foram apresentadas separadamente.

Foram considerados os registros nos idiomas inglês, espanhol e português; e documentos científicos publicados na forma de artigos originais ou relatórios de órgãos oficiais com a descrição do inquérito realizado. Artigos de revisão, editoriais, capítulos de livros, cartas, opiniões pessoais, comentários, resumos de congressos não foram considerados.

A restrição da faixa etária foi aplicada para permitir a comparabilidade com a Pesquisa Nacional de Saúde do Escolar (PeNSE) - Amostra 2, inquérito brasileiro realizado nos anos de 2009, 2012 e 2015 (OLIVEIRA et al., 2017). O limite inferior da data de publicação fixado em janeiro de 2014 foi definido visando a avaliação dos inquéritos com estimativas de prevalência de excesso de peso recentes.

Foram excluídos os artigos que: 1) reportavam estudos com abrangência regional/local; 2) apresentavam dados de população que não incluía a faixa etária entre 13 e 17 anos de ambos os sexos; 3) o artigo completo não estava disponível; 4) o idioma do texto completo era diferente de inglês, espanhol ou português; 5) apresentavam resultados de aplicações anteriores ao ano 2000 (no caso dos inquéritos realizados com frequência regular com publicações de aplicações mais recentes). Os artigos com divergências de classificação entre as duas revisoras foram resolvidos por consenso.

\section{Fontes de informação e estratégias de busca}

As estratégias de busca detalhadas foram realizadas nas seguintes bases de dados: MEDLINE (National Library of Medicine, Bethesda, MD), LILACS (Literatura Latino-Americana e do Caribe em Ciências da Saúde) e SciELO (Scientific Electronic Library On-line). Os descritores e suas combinações empregados na busca foram: ("health surveys" OR health survey") AND ("students") AND (“obesity" OR “overweight”). As buscas foram atualizadas até 11 de maio de 2019. Buscas adicionais foram realizadas diretamente no Google incluindo o nome ou sigla dos inquéritos encontrados e o termo "obesity". A lista de referência dos estudos incluídos foi revisada para avaliar a necessidade de incluir referências adicionais. 


\section{Seleção dos estudos e Extração dos dados}

As listas dos artigos identificados nas três bases de dados foram comparadas e, após a exclusão dos registros duplicados, foi feita uma seleção de artigos que atendiam os critérios de inclusão. Esta primeira triagem foi baseada na leitura dos títulos e resumos. Em uma segunda etapa, os revisores realizaram a leitura do artigo completo e excluíram aqueles que não atendiam os critérios de elegibilidade estabelecidos previamente.

As informações extraídas dos estudos foram: autor, ano de publicação, nome e ano de realização do inquérito, tamanho da amostra, faixa etária, prevalência de sobrepeso e/ou obesidade, critério adotado para classificação do estado nutricional, fatores avaliados para verificar associação com excesso de peso, definição desses fatores e os principais resultados do estudo.

\section{Avaliação da qualidade metodológica dos estudos incluídos}

Por se tratar de uma revisão sistemática que incluiu somente artigos que analisaram dados de inquéritos populacionais de base escolar, com representatividade nacional, não foi realizada avaliação da qualidade metodológica dos artigos baseada em critérios já consagrados. Isto porque reconhecidamente, os inquéritos populacionais, sejam eles domiciliares, telefônicos ou escolares, já contam com critérios metodológicos bastante rigorosos, que abarcam desde o processo de amostragem, a coleta dos dados propriamente dita até o processamento dos dados.

\section{Análise dos dados}

Os artigos foram agrupados segundo o inquérito a que se referiam e, para cada inquérito, as prevalências de sobrepeso e obesidade, com as definições adotadas, foram descritas, bem como os fatores associados a esse desfecho. Quando necessárias, buscas adicionais foram realizadas utilizando nome/sigla do inquérito ou mesmo as referências listadas nos artigos selecionados. 


\section{RESULTADOS}

Foram identificados 204 artigos distintos após a aplicação da estratégia de busca definida. 0 fluxograma com os detalhes do processo de seleção se encontra na Figura 1.

Figura 1. Fluxograma das fases de identificação, triagem e seleção de artigos sobre sobrepeso e obesidade em adolescentes, a partir de inquéritos populacionais de base escolar com representatividade nacional, publicados entre 2015 e 2019.
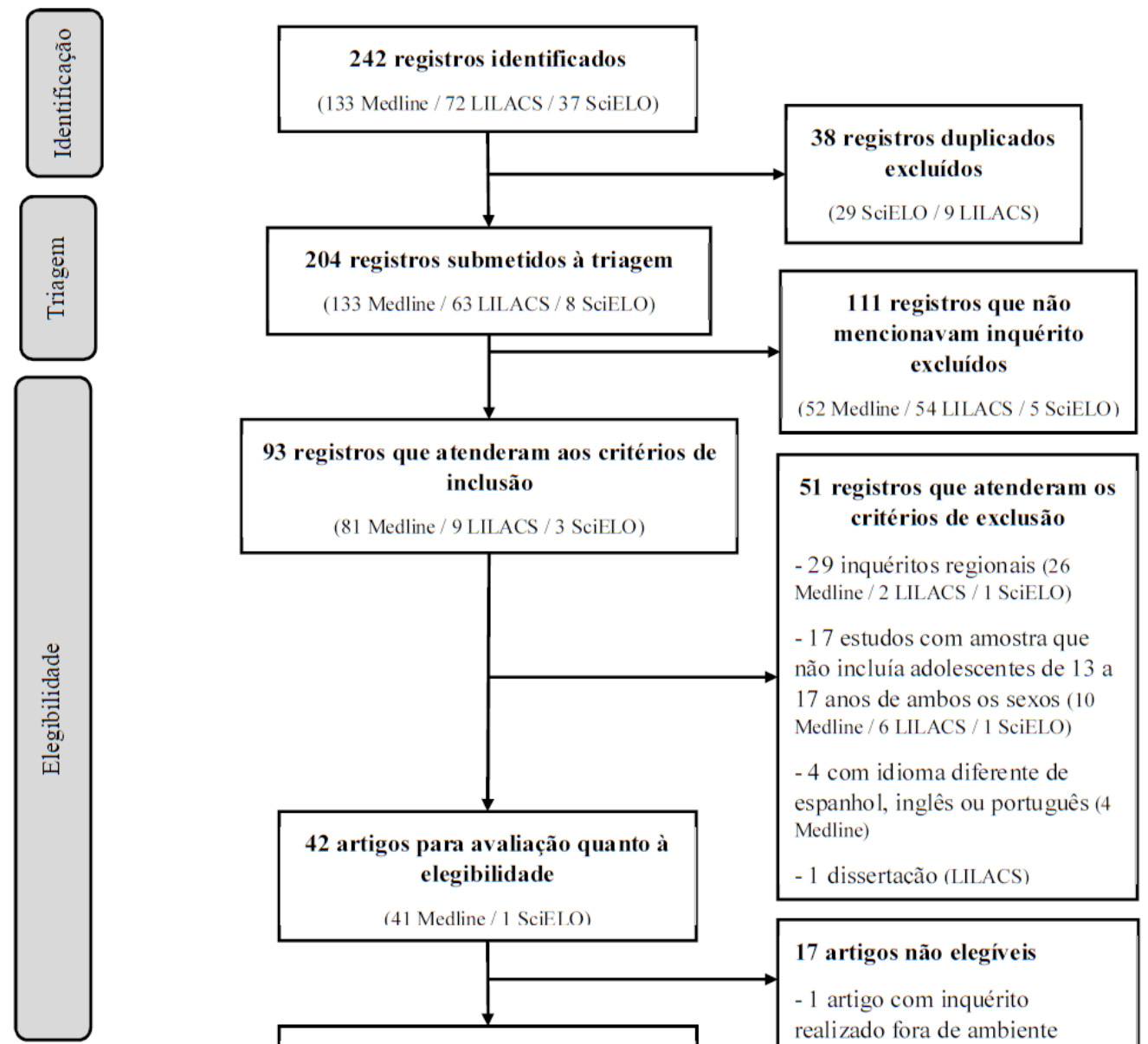

93 registros que atenderam aos critérios de inclusão

(81 Medline / 9 LILACS / 3 SciELO)

51 registros que atenderam os critérios de exclusão

- 29 inquéritos regionais (26 Medline/2 LILACS/ 1 SciELO)

- 17 estudos com amostra que não incluía adolescentes de 13 a

17 anos de ambos os sexos (10

Medline / 6 LILACS / 1 SciELO)

- 4 com idioma diferente de espanhol, inglês ou português (4 Medline)

- 1 dissertacão (LILACS)

17 artigos não elegíveis

- 1 artigo com inquérito realizado fora de ambiente escolar excluído

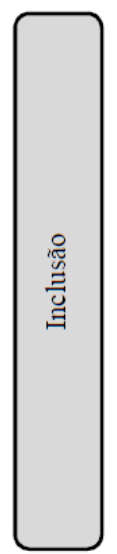

+4 artigos identificados nas referências ou pelo nome/site do inquérito

25 artigos elegíveis
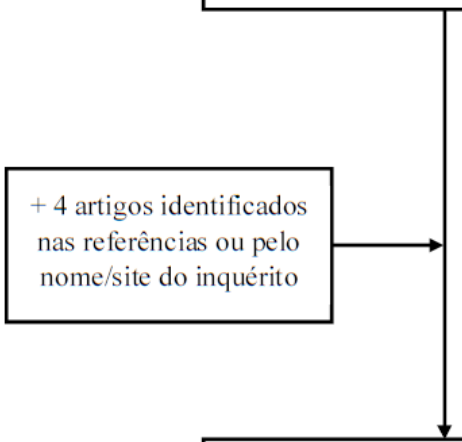

29 artigos incluídos na revisão
- 10 artigos que não relatam prevalência de excesso de peso

- 2 artigos com dados de

inquérito realizado antes do ano 2000

- 1 artigo sem as informações de delineamento amostral e data de realização

- 3 artigos que não foi possível acesso ao texto completo

Fonte: Própria. 
Entre os 29 artigos incluídos na revisão, foram analisados dados referentes a 11 diferentes inquéritos de base escolar, conduzidos entre os anos de 2007 e 2015, com delineamento e tamanho amostral adequados para garantir representatividade nacional, incluindo adolescentes de 13 a 17 anos (ou faixa etária mais abrangente) e que coletaram medidas antropométricas de peso e altura para o cálculo e categorização do índice de massa corporal (IMC) desses adolescentes. Os inquéritos identificados relataram dados de representatividade nacional para o Brasil, Estados Unidos, Canadá, China, Argentina, Filipinas, Camboja, Grécia e Irã, além de inquéritos que reuniram dados de vários países da Ásia e Europa e um global com países de baixa e média renda (Tabela 1).

Alguns dos inquéritos encontrados são realizados com periodicidade definida, como a PeNSE (Pesquisa Nacional de Saúde do Escolar), iniciada em 2009 e com previsão de ser realizada a cada 3 anos; o YBRSS (Youth Risk Behavior Surveillance System) e o CASPIAN (Childhood and Adolescence Surveillance and Prevention of Adult Non-communicable Disease) que têm periodicidade bianual, iniciados em 1990 e 2003, respectivamente. 
Tabela 1 - Inquéritos populacionais de representatividade nacional analisados nos artigos selecionados.

\begin{tabular}{|c|c|c|c|}
\hline & Autor, ano & Inquérito & Local/País \\
\hline 1 & Cureau et al., 2018 & $\begin{array}{l}\text { ERICA (Estudo dos Riscos Cardiovasculares em } \\
\text { Adolescentes)(CUREAU et al., 2018) }\end{array}$ & Brasil \\
\hline 2 & $\begin{array}{l}\text { Almeida et al., 2016; } \\
\text { Chaves et al., 2018; } \\
\text { Conde et al., } 2018\end{array}$ & $\begin{array}{l}\text { PeNSE (Pesquisa Nacional de Saúde do } \\
\text { Escolar)(ALMEIDA; ARAÚJO JÚNIOR, 2016; CHAVES et } \\
\text { al., 2018; CONDE et al., 2018) }\end{array}$ & Brasil \\
\hline 3 & $\begin{array}{l}\text { Kessaram et al., 2015; } \\
\text { Meldrum et al., 2014; } \\
\text { Sliwa et al., } 2017\end{array}$ & $\begin{array}{l}\text { YRBSS (Youth Risk Behavior Surveillance } \\
\text { System)(KESSARAM et al., 2015; MELDRUM; RESTIVO, } \\
\text { 2014; SLIWA et al., 2017) }\end{array}$ & $\begin{array}{l}\text { Ilhas do Pacífico* } \\
\text { Estados Unidos }\end{array}$ \\
\hline 4 & $\begin{array}{l}\text { Jayawardene et al., } \\
2014\end{array}$ & $\begin{array}{l}\text { NYPANS (National Youth Physical Activity and } \\
\text { Nutrition Study)(JAYAWARDENE; LOHRMANN; } \\
\text { YOUSSEFAGHA, 2014) }\end{array}$ & Estados Unidos \\
\hline 5 & Bookwala et al., 2014 & $\begin{array}{l}\text { YSS (Youth Smoking Survey)(BOOKWALA; ELTON- } \\
\text { MARSHALL; LEATHERDALE, 2014) }\end{array}$ & Canadá \\
\hline 6 & $\begin{array}{l}\text { Dong et al., 2018; } \\
\text { Song et al., } 2016\end{array}$ & $\begin{array}{l}\text { CNSSH (Chinese National Survey on Students' } \\
\text { Constitution and Health)(DONG et al., 2018; SONG et al., } \\
\text { 2016) }\end{array}$ & China \\
\hline 7 & Guo et al., 2019 & $\begin{array}{l}\text { NSCAHS (National School-based Chinese Adolescents } \\
\text { Health Survey)(GUO et al., 2019) }\end{array}$ & China \\
\hline 8 & $\begin{array}{l}\text { Ferrante et al., 2014; } \\
\text { Peltzer et al., 2015, } \\
\text { 2016; } \\
\text { Pengpid et al., 2016; }\end{array}$ & $\begin{array}{l}\text { GSHS (Global school-based Student Health } \\
\text { Survey)(FERRANTE et al., 2014; PELTZER; PENGPID, } \\
\text { 2015, 2016; PENGPID; PELTZER, 2016; YANG et al., 2019; } \\
\text { YEN et al., 2018) }\end{array}$ & $\begin{array}{l}\text { Filipinas, Ásia** } \\
\text { Ásia*** }\end{array}$ \\
\hline
\end{tabular}


Yang et al., 2019

Global $^{\S}$

Yen et al., 2018

Camboja

9 Arnaoutis et al., 2018

EYZHN (National Action for Children's

Health)(ARNAOUTIS et al., 2018)

Grécia

HBSC (Health Behaviors in School-Age

Estados Unidos ${ }^{\S \S}$

10

Children)(FARHAT; IANNOTTI; CACCAVALE, 2014;

Quick et al., 2014

QUICK et al., 2014)

Europa

Bahreynian et al.,

2015;

Heshmat et al., 2015;

Hovsepian et al.,

CASPIAN (Childhood and Adolescence Surveillance and

2017;

11 Jari et al., 2014;

Prevention of Adult Non- communicable

Kelishadi et al., 2018;

disease)(BAHREYNIAN et al., 2015; HESHMAT et al.,

República 2015; HOVSEPIAN et al., 2017; JARI et al., 2014;

Islâmica do Irã

KELISHADI et al., 2018; MOTLAGH et al., 2017;

Motlagh et al., 2017;

MOZAFARIAN et al., 2017; ZAHEDI et al., 2014)

Mozafarian et al.,

2017;

Zahedi et al., 2014

* Países e territórios das Ilhas do Pacífico: Guam, Ilhas Marianas Setentrionais, Palau, República das Ilhas Marshall, Samoa Americana

** Referente aos países (ano da pesquisa): Camboja (2013), Indonésia (2007), Malásia (2012), Myanmar (2007), Filipinas (2011), Tailândia (2008) e Vietnã (2013)

*** Referente aos países citados em ** acrescido de Brunei Darussalam (2014)

$\S 58$ países de baixa e média renda de 6 regiões definidas pela Organização Mundial da Saúde (África:

9; América - apenas Central, Sul e Caribe: 18; Mediterrâneo Oriental: 11; Europa: 1; Sudeste asiático:

6; Pacífico Ocidental: 12) e China.

§§ Inquérito comumente realizado em países da Europa, porém foi identificado na busca através de artigo que replicou o método em adolescentes dos EUA em 2005-06.

Fonte: Própria.

As prevalências de excesso de peso nos artigos analisados variaram desde 2,3\% no Camboja, país com alta proporção de desnutrição, até 36,8\% nos Estados Unidos. Houve 
grande variação nos fatores associados encontrados, visto que o objetivo da maioria dos estudos não era estimar a prevalência e/ou verificar os fatores associados. No entanto, foi possível identificar fatores associados ao excesso de peso como a privação do sono(MELDRUM; RESTIVO, 2014), deslocamento diário inativo até a escola (BOOKWALA; ELTON-MARSHALL; LEATHERDALE, 2014; PENGPID; PELTZER, 2016) e comportamento sedentário/alto tempo de tela (CUREAU et al., 2018; FARHAT; IANNOTTI; CACCAVALE, 2014; PELTZER; PENGPID, 2016). Notou-se também a inclusão do excesso de peso como covariável em estudos sobre saúde mental (GUO et al., 2019; JARI et al., 2014; YEN et al., 2018) (Tabela 2). 
Tabela 2 - Prevalência de excesso de peso em adolescentes a partir de inquéritos populacionais de base escolar com representatividade nacional.

\begin{tabular}{|c|c|c|c|c|c|c|c|}
\hline & Inquérito & Faixa etária & $\begin{array}{c}\text { Ano de } \\
\text { realização }\end{array}$ & $\begin{array}{c}\text { Tamanho } \\
\text { da } \\
\text { amostra }\end{array}$ & $\begin{array}{c}\text { Prevalência de } \\
\text { excesso de } \\
\text { peso* (\%) }\end{array}$ & $\begin{array}{l}\text { Critério para } \\
\text { classificação } \\
\text { do IMC** }\end{array}$ & Fatores associados / Comentários \\
\hline 1 & $\begin{array}{l}\text { ERICA (CUREAU et } \\
\text { al., 2018) }\end{array}$ & $12-17$ anos & 2013-14 & 62.063 & 25,8 & OMS & $\begin{array}{l}\text { O estudo avaliou a associação de várias combinações de } \\
\text { comportamentos não saudáveis com excesso de peso. } \\
\text { A combinação de inatividade física, alto tempo de tela e baixa } \\
\text { ingestão de fibras foi associada ao excesso de peso. } \\
\text { Fumo e consumo excessivo de álcool simultaneamente, apesar de } \\
\text { pouco frequente, apresentou associação com obesidade. }\end{array}$ \\
\hline 2 & $\begin{array}{l}\text { PeNSE (CHAVES et } \\
\text { al., 2018) }\end{array}$ & 5-19 anos & 2009 & 57.268 & 23,8 & OMS & $\begin{array}{l}\text { Consumo frequente de refrigerante foi associado a categorias mais } \\
\text { elevadas do Z-score do IMC apenas em adolescentes eutróficos do } \\
\text { sexo masculino. }\end{array}$ \\
\hline & $\begin{array}{l}\text { PeNSE (ALMEIDA; } \\
\text { ARAÚJO JÚNIOR, } \\
\text { 2016) }\end{array}$ & $13-15$ anos & 2012 & 66.844 & 15,8 & OMS & $\begin{array}{l}\text { O desfecho primário do estudo foi o atraso escolar, não o excesso de } \\
\text { peso. }\end{array}$ \\
\hline
\end{tabular}


PeNSE (CONDE et

al., 2018)

11-19 anos

2015

16.556

22,2

IOTF

Cor da pele branca, alunos de escolas particulares, da região sul, de área urbana e dos estratos socioeconômicos mais pobres apresentaram prevalências mais elevadas.

As prevalências foram mais elevadas no sexo feminino, especialmente após 15 anos de idade.

\begin{tabular}{|c|c|c|c|c|c|c|c|}
\hline 3 & $\begin{array}{l}\text { YRBSS (KESSARAM } \\
\text { et al., 2015) }\end{array}$ & $14-18$ anos & $2007-2013$ & - & $\begin{array}{l}24,4 \text { a } 60,7 \text { o' } \\
24,8 \text { a } 59,8 \text { ㅇ }\end{array}$ & OMS & $\begin{array}{l}\text { Reportou atividade física insuficiente e consumo excessivo de } \\
\text { refrigerante em vários países (sem análise de associação com } \\
\text { obesidade). A prevalência de obesidade variou de 12,5 a 40,4 nos } \\
\text { meninos e de } 11,5 \text { a } 37,3 \text { nas meninas. }\end{array}$ \\
\hline & $\begin{array}{l}\text { YRBSS (SLIWA et } \\
\text { al., 2017) }\end{array}$ & $14-18$ anos & 2013 & 23,173 & $\begin{array}{l}34,2 \\
14,5 \text { sobrepeso } \\
19,7 \text { obesidade }\end{array}$ & OMS & $\begin{array}{l}\text { Adolescentes com sobrepeso apresentaram menos chance de } \\
\text { consumir } \geq 1 \text { copo de leite por dia. Não foi encontrada associação } \\
\text { entre o consumo de leite e obesidade. } \\
\text { Não foi encontrada associação entre o consumo de suco natural e } \\
\text { excesso de peso. }\end{array}$ \\
\hline & $\begin{array}{l}\text { YRBSS (MELDRUM; } \\
\text { RESTIVO, 2014) }\end{array}$ & $14-18$ anos & 2011 & 14.285 & $13,0^{* * *}$ & OMS & $\begin{array}{l}\text { Foi verificada associação entre privação do sono ( } 5 \text { ou menos horas } \\
\text { de sono) e obesidade (aumento). }\end{array}$ \\
\hline 4 & $\begin{array}{c}\text { NYPANS } \\
\text { (JAYAWARDENE; } \\
\text { LOHRMANN; }\end{array}$ & $14-19$ anos & 2010 & 7.160 & $\begin{array}{l}\text { 18,1 sobrepeso } \\
18,7 \text { obesidade }\end{array}$ & OMS & O objetivo do estudo foi comparar peso e altura medidos e referidos. \\
\hline
\end{tabular}

Revista SUSTINERE, Rio de Janeiro, v. 9, n.1, p. 372-397, jan-jun, 2021 
Prevalência e fatores associados ao sobrepeso e obesidade em adolescentes

a partir de inquéritos populacionais de base escolar

YOUSSEFAGHA,

2014)

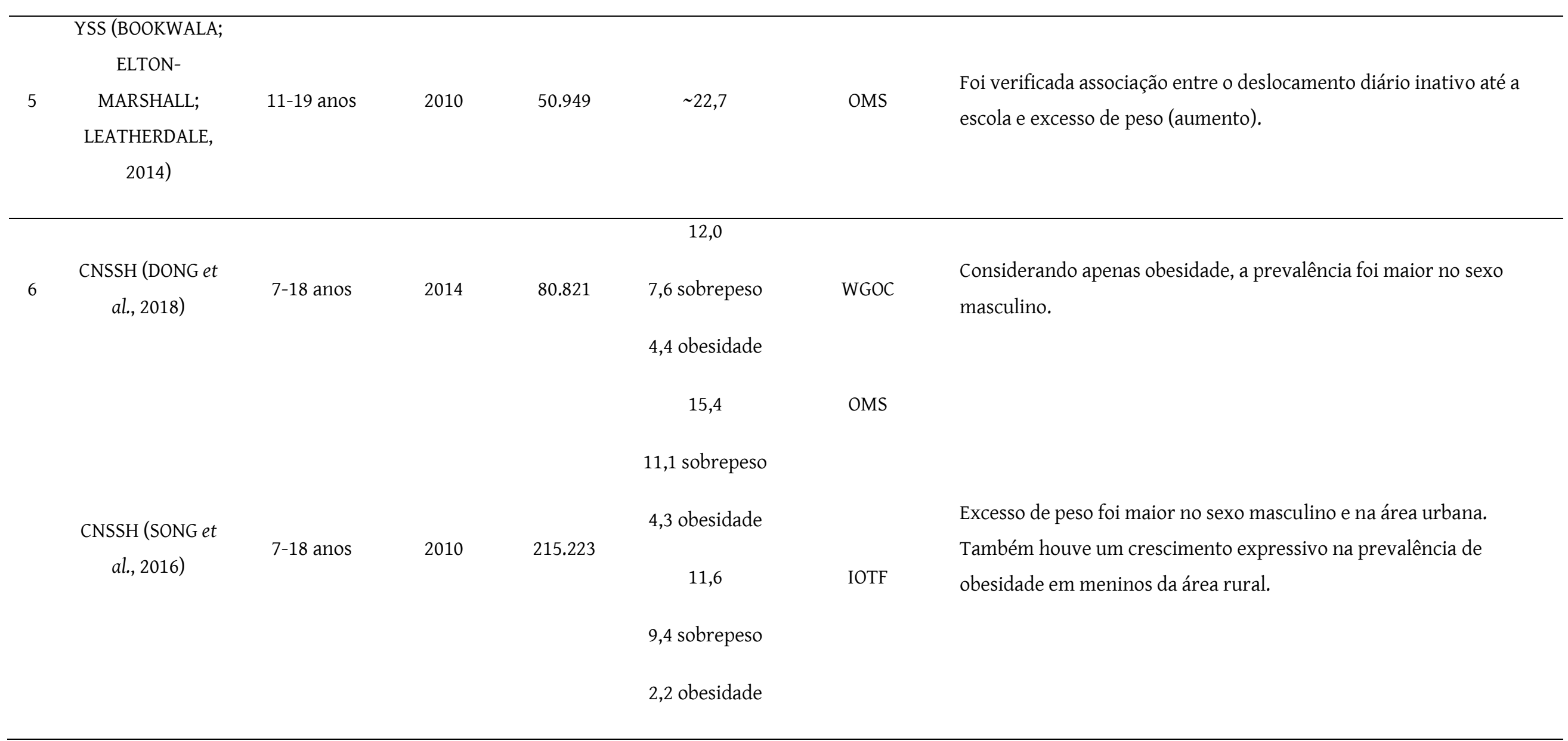


NSCAHS (GUO et

al., 2019)

$\sim 10-18$ anos

2015

\begin{abstract}
54.640
\end{abstract}
10,1 sobrepeso

WGOC

5,3 obesidade

\section{4,5}

et al., 2014)

13-15 anos

2012

20.697

28,6 sobrepeso

5,9 obesidade

\section{GSHS (PELTZER;}

PENGPID, 2016)

13-15 anos

2007-13

30.284

9,9

$9,70^{n}$

GSHS (PELTZER;

PENGPID, 2015)

$11-<16$ anos

2011

5.265

$9,4 ㅇ$

9,9

GSHS (PENGPID;

PELTZER, 2016)
13-15 anos

2007-13
GSHS (FERRANTE
O objetivo do estudo foi avaliar ideias suicidas. Foi relatado risco aumentado para meninos obesos e com baixo peso.
Aumento de $17 \%$ da prevalência de excesso de peso em relação a 2007 e maior no sexo masculino.

Foi verificada associação entre comportamento sedentário ( $\geq 3$ horas/dia sentado - fora da escola ou estudando).

Curva de percentil equivalente

Não houve associação com atividade física insuficiente $(<60$ minutos/dia de atividade física com intensidade moderada a intensa em pelo menos 5 dias/semana).

Queda de $44 \%$ e $21 \%$ da prevalência de excesso de peso em relação a 2003 para meninos e meninas, respectivamente.

equivalente

Prevalência de excesso de peso foi maior no sexo masculino, idade mais jovem (13 anos), proveniente de país com renda média-alta, em jovens que nunca passaram fome, e naqueles com trajeto diário para a escola inativo. percentil

equivalente 
Prevalência e fatores associados ao sobrepeso e obesidade em adolescentes a partir de inquéritos populacionais de base escolar

\section{0,4 obesidade \\ 36,1 (Brunei}

Darussalam)

18,4 sobrepeso

17,7 obesidade

\section{GSHS (YEN et al.}

2018)

11-18 anos

2012-13

3.806

17,3 sobrepeso

GSHS (YANG et al.,

2019)

12-15 anos

2007-13

117.325

8,6 obesidade

21,0

15,4 sobrepeso

Fatores adicionais associados à maior prevalência de excesso de peso: Meninos - consumo de 3+ porções de vegetais/dia e não ter amigos próximos / Meninas - consumo de fastfood 2+ vezes/semana, ser vítima de bullying e ter apoio dos colegas

Foi verificada maior prevalência de excesso de peso nos mais velhos, com maior consumo de fast-food, naqueles que relataram se sentir sozinho e que tentaram suicídio.

OMS-2

Sexo, consumo de frutas/vegetais, atividade física, fumo, uso de álcool, uso de drogas, ataques físicos, envolvimento em brigas e dificuldade para dormir não apresentaram associações significativas com excesso de peso.

OMS

Diferenças nas prevalências dos países/regiões.

Não foram encontradas diferenças nas prevalências por sexo e idade. 
5,6 obesidade

\section{EYZHN}

9 (ARNAOUTIS et al.,

6-18 anos

2015

2018)

\section{0,1}

,9 sobrepeso

8,2 obesidade
Estudo verificou que a presença de excesso de peso, baixa adesão à dieta Mediterrânea e tempo gasto em atividades sedentárias > 2 horas por dia foram positivamente associados com baixa aptidão física.

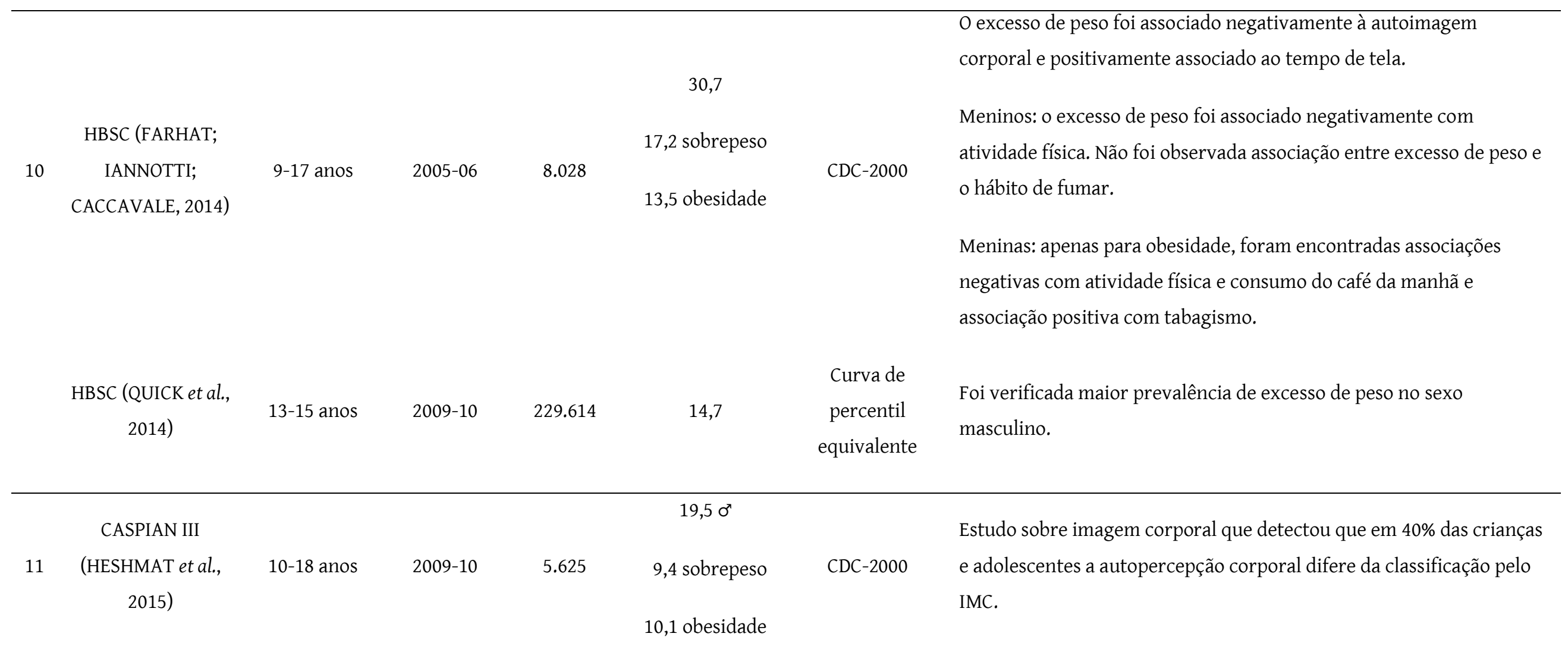

Revista SUSTINERE, Rio de Janeiro, v. 9, n.1, p. 372-397, jan-jun, 2021 
8,0 sobrepeso

8,9 obesidade

16,7

\section{CASPIAN III (JARI}

et al., 2014)

10-18 anos

$2009-10$

5.528

\section{7,9 sobrepeso}

8,8 obesidade

CASPIAN IV

(BAHREYNIAN $e$

al., 2015)

6-18 anos

2011-12

13.486

9,5 sobrepeso

12,0 obesidade

\section{CASPIAN IV}

(HOVSEPIAN et al., 2017)

CASPIAN IV

(MOZAFARIAN et

al., 2017)
6-18 anos

$2011-12$

23.043

17,8

20,3

6-18 anos

$2011-12$

13.486
Estudo mostrou que o excesso de peso não apresentou associação com depressão, ansiedade e insônia em adolescentes iranianos.

Estudo verificou os fatores associados à adesão a dieta de redução de peso por crianças e adolescentes e verificou que o excesso de peso, tanto medido pela classificação do IMC quanto auto percebido aumenta a chance dessa adesão.

Estudo sobre qualidade de vida relacionada à saúde encontrou escores mais baixos de qualidade de vida nos adolescentes obesos maiores de 12 anos. Também foi verificada associação inversa significativa do escore total e do domínio psicossocial com sobrepeso e obesidade.

Foi verificada associação do excesso de peso com assistir TV >2 horas por dia. 
10,2 obesidade

CASPIAN IV

(ZAHEDI et al. 2014)

CASPIAN V

(KELISHADI et al. 2018)

CASPIAN V

(MOTLAGH et al. 2017)
6-18 anos

2011-12

13.486

9,7 sobrepeso

Não informado

11,9 obesidade

20,9

,4 sobrepeso

11,5 obesidade

20,8

9,4 sobrepeso

11,4 obesidade
Não houve associação com uso de computador $>2$ horas por dia, nem tempo de tela $>4$ horas por dia.

O objetivo do estudo foi explorar a associação entre o consumo de junkfood e a saúde mental de crianças e adolescentes. Foi encontrada associação significativa entre o consumo de junk food e problemas de saúde mental, independente do índice de massa corporal, tempo de tela, nível socioeconômico, atividade física e história familiar.

Estudo verificou associação entre ser filho(a) único(a) e fatores de risco cardio-metabólico. Crianças/adolescentes filhos(as) únicos(as) tiveram maior chance de apresentarem obesidade abdominal.

Estudo apresentou a prevalência de alguns comportamentos e fatores de risco relacionados à saúde. Aponta que o consumo de junk food, atividades de lazer sedentárias e prevalência relativamente alta de excesso de peso e dislipidemia merecem mais atenção.

IMC: índice de massa corporal IC95\%: Intervalo de Confiança de 95\%

$\sigma^{x}:$ meninos

O: meninas

* Excesso de peso = sobrepeso + obesidade

** CDC-2000: Pontos de corte da curva de IMC por idade e sexo propostos pelo Centers for Disease Control and Prevention no ano 2000

Curva de percentil equivalente: International Centile curves propostas por Cole, TJ et al. (2000)(COLE, 2000)

IOTF: Pontos de corte da curva de IMC por idade e sexo propostos pela International Obesity Task Force

OMS: Pontos de corte da curva de IMC por idade e sexo propostos pela Organização Mundial da Saúde

OMS-2: Pontos de corte de IMC propostos pela Organização Mundial da Saúde para adultos (Sobrepeso: IMC $\geq 25 \mathrm{~kg} / \mathrm{m}^{2}$ )

WGOC: Pontos de corte da curva de IMC por idade e sexo propostos pelo Working Group on Obesity in China

*** Referente a obesidade apenas.

Fonte: Própria. 


\section{DISCUSSÃO}

Os resultados da busca dessa revisão sistemática identificaram 11 inquéritos populacionais de base escolar com representatividade nacional, realizados em países com características muito diferentes entre si. Dada a importância do inquérito americano YRBSS, iniciado em 1990, o mesmo foi utilizado como referência para o delineamento dos inquéritos realizados posteriormente, a exemplo do Global school-based Student Health Survey (GSHS) que atualmente é utilizado como padrão em vários países e, nacionalmente, a PeNSE, que conta com três edições, e descreve as condições de saúde dos escolares brasileiros.

Após revisão dos resultados, vimos que na grande maioria dos países as prevalências de excesso de peso em adolescentes se encontram entre $10 \%$ e $20 \%$, embora haja muita variação em função da faixa etária estudada e critérios adotados para a definição de sobrepeso e obesidade. Notou-se também que os estudos com o objetivo principal de estimar a prevalência são menos frequentes (CONDE et al., 2018; KESSARAM et al., 2015; SONG et al., 2016; YANG et al., 2019) e mais estudos estão sendo realizados a fim de avaliar os fatores associados ao excesso de peso (CHAVES et al., 2018; CUREAU et al., 2018; FERRANTE et al., 2014; GUO et al., 2019; HOVSEPIAN et al., 2017; JARI et al., 2014; PENGPID; PELTZER, 2016; YEN et al., 2018), ou incluindo o excesso de peso como variável de ajuste em análises de fatores associados a outras doenças (ALMEIDA; ARAÚJO JÚNIOR, 2016; DONG et al., 2018; MELDRUM; RESTIVO, 2014; ZAHEDI et al., 2014).

Houve uma variação nos resultados da prevalência de excesso de peso de acordo com a situação econômica do país, sendo esta menor nos países mais pobres e maior em países mais ricos (YANG et al., 2019; YEN et al., 2018). Tais achados corroboram os resultados de Abarca-Gómez e cols. (2017) que revelam uma estagnação da prevalência, em valores elevados, principalmente em países de alta renda, e um aumento em países menos desenvolvidos economicamente (NCD RISK FACTOR COLLABORATION (NCD-RISC), 2017). Isso nos leva a supor que o acesso a uma diversidade maior de alimentos industrializados associada às novas tecnologias disponíveis e acessíveis aos adolescentes nos países mais ricos contribuíram para um comportamento mais sedentário e, consequentemente, para o excesso de peso desses adolescentes. À medida que esse acesso alcança os adolescentes que vivem em países menos desenvolvidos, observa-se um aumento nas prevalências de excesso de peso. 
Dentre os fatores associados ao excesso de peso, os fatores modificáveis, como a prática de atividades físicas e hábitos alimentares saudáveis, têm uma grande contribuição para o desfecho. Medidas fiscais como a taxação de alimentos considerados não saudáveis e a desoneração daqueles saudáveis, associadas a programas de educação física adequados e mais atrativos no âmbito escolar, são exemplos de intervenções que poderiam contribuir para a adoção de hábitos de vida mais saudáveis já na infância.

Outro fator relevante associado ao excesso de peso está relacionado à saúde mental, tendo em vista que vários estudos identificaram associações entre sentir-se sozinho (PENGPID; PELTZER, 2016), ter ideias suicidas (GUO et al., 2019; YEN et al., 2018), pior qualidade de vida(HOVSEPIAN et al., 2017), sofrer bullying(PENGPID; PELTZER, 2016) e não ter amigos próximos (PENGPID; PELTZER, 2016).

Como limitação desse estudo, podemos citar a restrição na busca por inquéritos epidemiológicos a partir dos termos "health surveys" ou "health survey". Verificou-se que nem sempre os artigos tinham um desses termos no título ou resumo, limitando a inclusão de referências. Sugere-se a inclusão da sigla ou nome do inquérito no título do artigo e o tipo de estudo nas palavras-chave, padronização que poderia ser seguida nas publicações, na tentativa de facilitar a busca em processos de revisões sistemáticas e meta-análises. Além disso, alguns dos artigos encontrados que utilizaram dados de inquérito nacional de base escolar não apresentavam valores de prevalência de excesso de peso, apenas análises que utilizaram a variável IMC de forma contínua, portanto, não foram incluídos nesta revisão.

A partir desta revisão foi possível verificar a relevância da realização de inquéritos populacionais de base escolar com abrangência nacional, que já ocorrem em muitos países e possibilitam a obtenção de informações que refletem a complexidade e a dinâmica de mudanças a que está sujeito esse grupo populacional. A análise e acompanhamento dos resultados desses inquéritos é uma valiosa fonte de informação tanto para os gestores da área da saúde quanto para a área da educação, pois auxiliam na identificação de fatores de risco modificáveis para diversas doenças, contribuindo na elaboração de propostas de intervenções mais efetivas, especialmente se mantidas a temporalidade de realização e a comparabilidade desses inquéritos com dados de outros países. 


\section{CONCLUSÃO}

Após a revisão dos resultados, mesmo com algumas limitações nas restrições de busca como já citado, conclui-se que uma excelente ferramenta de avaliação das condições de saúde de crianças e adolescentes é a utilização de inquéritos populacionais de base escolar associado à análise e acompanhamento dos resultados, com identificação de fatores de risco modificáveis relevantes para diversas doenças, e assim elaboração de propostas de intervenção mais efetivas.

Nos levantamentos desse estudo, foi de indubitável importância a utilização desses inquéritos populacionais de base escolar com abrangência nacional por possibilitar o encontro e análise comparativa dos fatores de risco para o excesso de peso em crianças e adolescente, somada a informações que refletem a complexidade de mudanças a que está sujeito esse grupo social, especialmente se mantidas novas revisões seguindo a temporalidade desses inquéritos nacionais.

\section{REFERÊNCIAS}

ALMEIDA, Aléssio Tony Cavalcanti de; ARAÚJO JÚNIOR, Ignácio Tavares de. Efeitos da exposição aos fatores de risco comportamentais à saúde sobre o atraso escolar no Brasil. Revista Brasileira de Economia, v. 70, n. 2, p. 129-169, 2016. Disponível em: <https://doi.org/10.5935/0034-7140.20160007>. Acesso em: 28 de maio de 2019.

ARNAOUTIS, Giannis; GEORGOULIS, Michael; PSARRA, Glykeria; MILKONIDOU, Anna; PANAGIOTAKOS, Demosthenes B.; KYRIAKOU, Dafni; BELLOU, Elena; TAMBALIS, Konstantinos D.; SIDOSSIS, Labros S. Association of anthropometric and lifestyle parameters with fitness levels in Greek Schoolchildren: Results from the EYZHN Program. Frontiers in Nutrition, v. 5, p. 1-10, 2018. Disponível em: <https://doi.org/10.3389/fnut.2018.00010>. Acesso em: 30 de maio de 2019.

BAHREYNIAN, Maryam; QORBANI, Mostafa; MOTLAGH, Mohammad Esmaeil; HESHMAT, Ramin; ARDALAN, Gelayol; KELISHADI, Roya. Association of perceived weight status versus body mass index on adherence to weight-modifying plan among Iranian children and adolescents: The CASPIAN-IV Study. Indian pediatrics, India, v. 52, n. 10, p. 857-863, 2015. Disponível em: <https://doi.org/10.1007/s13312-015-0732-9>. Acesso em: 27 de junho de 2019.

BOOKWALA, Ammar; ELTON-MARSHALL, Tara; LEATHERDALE, Scott T. Factors associated with active commuting among a nationally representative sample of Canadian youth. Canadian journal of public health, Switzerland, v. 105, n. 5, p. e348-53, Jul. 2014. Disponível em: <http://www.ncbi.nlm.nih.gov/pubmed/25365269>. Acesso em: 27 de junho de 2019.

BRASIL; MINISTÉRIO DA SAÚDE; SECRETARIA DE VIGILÂNCIA EM SAÚDE; DEPARTAMENTO DE VIGILÂNCIA DE DOENÇAS E AGRAVOS NÃO TRANSMISSÍVEIS E PROMOÇÃO DA SAÚDE. [Vigitel Brazil 2017: surveillance of risk and protective factors for chronic diseases by telephone survey: estimates of frequency and sociodemographic 
distribution of risk and protective factors for chronic diseases in the capitals of the 26 Brazilian st. 2018. Disponível em: $<$ http://bvsms.saude.gov.br/bvs/publicacoes/vigitel_brasil_2017_vigilancia_fatores_riscos.pdf>. Acesso em: 23 de agosto de 2018.

CENTERS FOR DISEASE CONTROL AND PREVENTION (CDC). Behavioral Risk Factor Surveillance System - BRFSS. 2018. March 22, 2018. Disponível em: <https://www.cdc.gov/brfss/>. Acesso em: 27 de março de 2018.

CENTERS FOR DISEASE CONTROL AND PREVENTION (CDC). Youth Risk Behavior Surveillance System (YRBSS) Overview. 2019. CDC. Disponível em: <https://www.cdc.gov/healthyyouth/data/yrbs/overview.htm>. Acesso em: 11 de março de 2019.

CHAVES, Otaviana Cardoso; VELASQUEZ-MELENDEZ, Gustavo; COSTA, Dario Alves da Silva; CAIAFFA, Waleska Teixeira. Soft drink consumption and body mass index in Brazilian adolescents: National Adolescent Student Health Survey. Revista brasileira de epidemiologia, Brazil, v. 21, n. suppl 1, p. e180010, 2018. Disponível em: <https://doi.org/10.1590/1980-549720180010.supl.1>. Acesso em: 13 de julho de 2019.

COLE, T. J.; LOBSTEIN, T. Extended international (IOTF) body mass index cut-offs for thinness, overweight and obesity. Pediatric Obesity, v. 7, n. 4, p. 284-294, 2012. Disponível em: <https://doi.org/10.1111/j.20476310.2012.00064.X>. Acesso em: 27 de junho de 2019.

COLE, T. J. Establishing a standard definition for child overweight and obesity worldwide: international survey. BMJ, v. 320, n. 7244, p. 1240-1240, 2000. Disponível em: <https://doi.org/10.1136/bmj.320.7244.1240>. Acesso em: 27 de junho de 2019.

CONDE, Wolney Lisbôa; MAZZETI, Camila Medeiros da Silva; SILVA, Jéssica Cumpian; SANTOS, Iolanda Karla Santana dos; SANTOS, Aline Micaele dos Reis. Estado nutricional de escolares adolescentes no Brasil: a Pesquisa Nacional de Saúde dos Escolares 2015. Revista Brasileira de Epidemiologia, v. 21, n. suppl 1, p. 1-12, 2018. Disponível em: <https://doi.org/10.1590/1980-549720180008.supl.1>. Acesso em: 28 de maio de 2019.

CUREAU, F V; SPARRENBERGER, K; BLOCH, K V; EKELUND, U; SCHAAN, B D. Associations of multiple unhealthy lifestyle behaviors with overweight/obesity and abdominal obesity among Brazilian adolescents: A countrywide survey. Nutrition, metabolism, and cardiovascular diseases : NMCD, Netherlands, v. 28, n. 7, p. 765774, 2018. Disponível em: <https://doi.org/10.1016/j.numecd.2018.04.012>. Acesso em: 27 de junho de 2019.

DANIELS, S. R.; HASSINK, S. G. The Role of the Pediatrician in Primary Prevention of Obesity. PEDIATRICS, v. 136, n. 1, p. e275-e292, 1 Jul. 2015. Disponível em: <https://doi.org/10.1542/peds.2015-1558>. Acesso em: 11 de abril de 2018.

DONG, Yanhui; ZOU, Zhiyong; YANG, Zhaogeng; WANG, Zhenghe; YANG, Yide; MA, Jun; DONG, Bin; MA, Yinghua; ARNOLD, Luke. Prevalence of excess body weight and underweight among 26 Chinese ethnic minority children and adolescents in 2014: a cross-sectional observational study. BMC public health, England, v. 18, n. 1, p. 562, 2018. Disponível em: <https://doi.org/10.1186/s12889-018-5352-6>. Acesso em: 27 de junho de 2019.

FARHAT, Tilda; IANNOTTI, Ronald J; CACCAVALE, Laura J. Adolescent overweight, obesity and chronic diseaserelated health practices: mediation by body image. Obesity facts, Switzerland, v. 7, n. 1, p. 1-14, 2014. Disponível em: <https://doi.org/10.1159/000357601>. Acesso em: 27 de junho de 2019.

FERRANTE, Daniel; LINETZKY, Bruno; PONCE, Miguel; GOLDBERG, Lucila; KONFINO, Jonathan; LASPIUR, Sebastian. Prevalence of overweight, obesity, physical activity and tobacco use in Argentine youth: Global School-Based Student Health Survey and Global Youth Tobacco Survey, 2007-2012. Archivos argentinos de pediatria, Argentina, v. 112, n. 6, p. 496-503, 2014. Disponível em: <https://doi.org/10.5546/aap.2014.496>. Acesso em: 27 de junho de 2019.

FUNDAÇÃO NACIONAL DE SAÚDE. Guia de vigilância epidemiológica. 5th ed. Brasília: Ministério da Saúde, 2002. Disponível em: $<$ http://bvsms.saude.gov.br/bvs/publicacoes/funasa/guia_vig_epi_vol_l.pdf>. Acesso em: 18 de novembro de 2017.

GALVÃO, Taís Freire; PANSANI, Thais de Souza Andrade; HARRAD, David; (TRADUTORES). Principais itens para relatar Revisões sistemáticas e Meta-análises: A recomendação PRISMA. Epidemiologia e Serviços de Saúde, 
v. 24, n. 2, p. 335-342, 2015. Disponível em: <https://doi.org/10.5123/S1679-49742015000200017>. Acesso em: 28 de maio de 2018.

GUO, Lan; XU, Yan; HUANG, Guoliang; GAO, Xue; DENG, Xueqing; LUO, Min; XI, Chuhao; ZHANG, Wei-Hong; LU, Ciyong. Association between body weight status and suicidal ideation among Chinese adolescents: the moderating role of the child's sex. Social psychiatry and psychiatric epidemiology, Germany, 2019. Disponível em: <https://doi.org/10.1007/s00127-019-01661-6>. Acesso em: 27 de junho de 2019.

HESHMAT, Ramin; KELISHADI, Roya; MOTAMED-GORJI, Nazgol; MOTLAGH, Mohammad-Esmaeil; ARDALAN, Gelayol; ARIFIRAD, Tahereh; RASTAD, Hadith; ASAYESH, Hamid; DJALALINIA, Shirin; LARIJANI, Bagher; QORBANI, Mostafa. Association between body mass index and perceived weight status with self-rated health and life satisfaction in Iranian children and adolescents: the CASPIAN-III study. Quality of life research : an international journal of quality of life aspects of treatment, care and rehabilitation, Netherlands, v. 24, n. 1, p. 263-272, 2015. Disponível em: < https://doi.org/10.1007/s11136-014-0757-X>. Acesso em: 28 de maio de 2018.

HOVSEPIAN, Silva; QORBANI, Mostafa; MOTLAGH, Mohammad Esmaeil; MADADY, Aryan; MANSOURIAN, Morteza; GORABI, Armita Mahdavi; KELISHADI, Roya. Association of obesity and health related quality of life in Iranian children and adolescents: the Weight Disorders Survey of the CASPIAN-IV study. Journal of pediatric endocrinology \& metabolism: JPEM, Germany, v. 30, n. 9, p. 923-929, 2017. Disponível em: $<$ https://doi.org/10.1515/jpem-2016-0402>. Acesso em: 27 de junho de 2019.

HULLEY, Stephen B.; CUMMINGS, Steven R.; BROWNER, Warren S.; GRADY, Deborah G.; NEWMAN, Thomas B. Delineando a pesquisa clínica. 400 p., $4^{\mathrm{a}}$ ed. Artmed, 2015.

IANNOTTI, Ronald J. Health Behavior in School-Aged Children (HBSC), 2009-2010. 2013. Inter-university Consortium for Political and Social Research. Disponível em: <https://doi.org/10.3886/ICPSR34792.v1>. Acesso em: 27 de setembro de 2017.

IBGE; COORDENAÇÃO DE POPULAÇÃO E INDICADORES SOCIAIS. Pesquisa nacional de saúde do escolar: 2015. Rio de Janeiro: [s. n.], 2016. Disponível em: <https://biblioteca.ibge.gov.br/visualizacao/livros/liv97870.pdf>. Acesso em: 22 de fevereiro de 2019.

JARI, Mohsen; QORBANI, Mostafa; MOTLAGH, Mohammad Esmaeil; HESHMAT, Ramin; ARDALAN, Gelayol; KELISHADI, Roya. Association of overweight and obesity with mental distress in Iranian adolescents: The CASPIAN-III Study. International journal of preventive medicine, Iran, v. 5, n. 3, p. 256-261, 2014. Disponível em: <https://www.ncbi.nlm.nih.gov/pmc/articles/PMC4018633/>. Acesso em: 27 de junho de 2019.

JAYAWARDENE, Wasantha; LOHRMANN, David; YOUSSEFAGHA, Ahmed. Discrepant body mass index: behaviors associated with height and weight misreporting among US adolescents from the National Youth Physical Activity and Nutrition Study. Childhood obesity (Print), United States, v. 10, n. 3, p. 225-233, 2014. Disponível em: <https://doi.org/10.1089/chi.2014.0002>. Acesso em: 27 de junho de 2019.

JI, C.-Y. The prevalence of childhood overweight/obesity and the epidemic changes in 1985-2000 for Chinese school-age children and adolescents. Obesity Reviews, v. 9, n. s1, p. 78-81, 2008. Disponível em: < https://doi.org/10.1111/j.1467-789X.2007.00443.X >. Acesso em: 25 de fevereiro de 2019.

KELISHADI, Roya; QORBANI, Mostafa; REZAEI, Fatemeh; MOTLAGH, Mohammad Esmaeil; DJALALINIA, Shirin; ZIAODINI, Hasan; TAHERI, Majzoubeh; OCHI, Fatemeh; SHAFIEE, Gita; AMINAEI, Tahereh; MAHDAVI GORABI, Armita; HESHMAT, Ramin. Is single-child family associated with cardio-metabolic risk factors: the CASPIAN-V study. BMC cardiovascular disorders, England, v. 18, n. 1, p. 109, 2018. Disponível em: $<$ https://doi.org/10.1186/s12872-018-0844-y>. Acesso em: 27 de junho de 2019.

KESSARAM, Tara; MCKENZIE, Jeanie; GIRIN, Natalie; MERILLES, Onofre Edwin A Jr; PULLAR, Jessica; ROTH, Adam; WHITE, Paul; HOY, Damian. Overweight, obesity, physical activity and sugar-sweetened beverage consumption in adolescents of Pacific islands: results from the Global School-Based Student Health Survey and the Youth Risk Behavior Surveillance System. BMC obesity, England, v. 2, p. 34, 2015. Disponível em: <https://doi.org/10.1186/s40608-015-0062-4>. Acesso em: 27 de junho de 2019. 
KIM, Yoonjung; CHOI, Sunhye; CHUN, Chaemin; PARK, Suyeon; KHANG, Young-Ho; OH, Kyungwon. Data Resource Profile: The Korea Youth Risk Behavior Web-based Survey (KYRBS). International Journal of Epidemiology, v. 45, p.1076-1076e, 2016. Disponível em: <https://doi.org/10.1093/ije/dyw070>. Acesso em: 11 de maio de 2019.

KUCZMARSKI, Robert J; OGDEN, Cynthia L; GUO, Shumei S; GRUMMER-STRAWN, Laurence M; FLEGAL, Katherine M; MEI, Zuguo; WEI, Rong; CURTIN, Lester R; ROCHE, Alex F; JOHNSON, Clifford L. 2000 CDC Growth Charts for the United States: methods and development. Vital and health statistics, v. 11, n. 246, p. 1-190, 2002. Disponível em: < https://pubmed.ncbi.nlm.nih.gov/12043359/ >. Acesso em: 30 de outubro de 2019.

MELDRUM, Ryan Charles; RESTIVO, Emily. The behavioral and health consequences of sleep deprivation among U.S. high school students: relative deprivation matters. Preventive medicine, United States, v. 63, p. 24-28, 2014. Disponível em: <https://doi.org/10.1016/j.ypmed.2014.03.006>. Acesso em: 27 de junho de 2019.

MITCHELL, Nia S.; CATENACCI, Victoria A.; WYATT, Holly R.; HILL, James O. Obesity: Overview of an Epidemic. Psychiatric Clinics of North America, v. 34, n. 4, p. 717-732, 2011. Disponível em: <https://doi.org/10.1016/j.psc.2011.08.005 >. Acesso em: 28 de maio de 2018.

MOTLAGH, Mohammad Esmaeil; ZIAODINI, Hasan; QORBANI, Mostafa; TAHERI, Majzoubeh; AMINAEI, Tahereh; GOODARZI, Azam; ATAIE-JAFARI, Asal; REZAEI, Fatemeh; AHADI, Zeinab; SHAFIEE, Gita; SHAHSAVARI, Ali; HESHMAT, Ramin; KELISHADI, Roya. Methodology and early findings of the fifth survey of childhood and adolescence surveillance and prevention of adult noncommunicable disease: The CASPIAN-V Study. International journal of preventive medicine, Iran, v. 8, p. 4, 2017. Disponível em: < https://doi.org/10.4103/2008-7802.198915>. Acesso em: 27 de junho de 2019.

MOZAFARIAN, Nafiseh; MOTLAGH, Mohammad Esmaeil; HESHMAT, Ramin; KARIMI, Siavash; MANSOURIAN, Morteza; MOHEBPOUR, Fatemeh; QORBANI, Mostafa; KELISHADI, Roya. Factors associated with screen time in Iranian children and adolescents: The CASPIAN-IV Study. International journal of preventive medicine, Iran, v. 8, p. 31, 2017. Disponível em: <https://doi.org/10.4103/ijpvm.IJPVM_36_17>. Acesso em: 27 de junho de 2019.

NCD RISK FACTOR COLLABORATION (NCD-RISC). Worldwide trends in body-mass index, underweight, overweight, and obesity from 1975 to 2016: a pooled analysis of 2416 population-based measurement studies in 128.9 million children, adolescents, and adults. The Lancet, v. 390, n. 10113, p. 2627-2642, 2017. Disponível em: <https://doi.org/10.1016/S0140-6736(17)32129-3>. Acesso em: 22 de fevereiro de 2019.

OLIVEIRA, Max Moura de; CAMPOS, Maryane Oliveira; ANDREAZZI, Marco Antonio Ratzsch de; MALTA, Deborah Carvalho. Características da Pesquisa Nacional de Saúde do Escolar - PeNSE. Epidemiologia e Serviços de Saúde, v. 26, n. 3, p. 605-616, 2017. Disponível em: <https://doi.org/10.5123/S1679-49742017000300017>. Acesso em: 28 de maio de 2019.

PELTZER, Karl; PENGPID, Supa. Health risk behaviour among in-school adolescents in the Philippines: Trends between 2003, 2007 and 2011, A Cross-Sectional Study. International journal of environmental research and public health, Switzerland, v. 13, n. 1, p. 73, 2015. Disponível em: <https://doi.org/10.3390/ijerph13010073>. Acesso em: 27 de junho de 2019.

PELTZER, Karl; PENGPID, Supa. Leisure time physical inactivity and sedentary behaviour and lifestyle correlates among students aged 13-15 in the Association of Southeast Asian Nations (ASEAN) Member States, 2007-2013. International journal of environmental research and public health, Switzerland, v. 13, n. 2, p. $217,2016$. Disponível em: <https://doi.org/10.3390/ijerph13020217>. Acesso em: 27 de junho de 2019.

PENGPID, Supa; PELTZER, Karl. Overweight, obesity and associated factors among 13-15 years old students in the Association of Southeast Asian Nations Member countries, 2007-2014. The Southeast Asian journal of tropical medicine and public health, Thailand, v. 47, n. 2, p. 250-262, 2016. Disponível em: <https://pubmed.ncbi.nlm.nih.gov/27244964/>. Acesso em: 27 de junho de 2019.

QUICK, V.; NANSEL, T. R.; LIU, D.; LIPSKY, L. M.; DUE, P.; IANNOTTI, R. J. Body size perception and weight control in youth: 9-year international trends from 24 countries. International Journal of Obesity, v. 38, n. 7, p. 988994, 2014. Disponível em: <https://doi.org/10.1038/ijo.2014.62>. Acesso em: 22 de fevereiro de 2019. 
SLIWA, Sarah A; MILLER, Gabrielle F; BRENER, Nancy D; PARK, Sohyun; MERLO, Caitlin L. District Policies and Practices Vary in Their Association With Adolescents' Consumption of Milk and 100\% Fruit Juice. The Journal of adolescent health : official publication of the Society for Adolescent Medicine, United States, v. 60, n. 5, p. 577-583, 2017. Disponível em: <https://doi.org/10.1016/j.jadohealth.2016.11.021>. Acesso em: 27 de junho de 2019.

SOCIEDADE BRASILEIRA DE PEDIATRIA; DEPARTAMENTO CIENTÍFICO DE NUTROLOGIA. Obesidade na infância e adolescência - Manual de Orientação. $[S$. l.: s. n.], 2012 . Disponível em: <https://www.sbp.com.br/imprensa/detalhe/nid/manual-de-orientacao-sobre-obesidade-na-infancia-eadolescencia-esta-disponivel-para-os-associados-da-sbp/>. Acesso em: 30 de novembro de 2019.

SONG, Yi; WANG, Hai-Jun; DONG, Bin; MA, Jun; WANG, Zhiqiang; AGARDH, Anette. 25-year trends in gender disparity for obesity and overweight by using WHO and IOTF definitions among Chinese school-aged children: a multiple cross-sectional study. BMJ Open, v. 6, n. 9, p. e011904, 2016. Disponível em: <https://doi.org/10.1136/bmjopen-2016-011904>. Acesso em: 22 de fevereiro de 2019.

SZWARCWALD, Célia Landmann; DAMACENA, Giseli Nogueira. Amostras complexas em inquéritos populacionais: planejamento e implicações na análise estatística dos dados. Revista Brasileira de Epidemiologia, v. 11, n. suppl 1, p. 38-45, 2008. Disponível em: <https://doi.org/10.1590/S1415790X2008000500004>. Acesso em: 22 de fevereiro de 2019.

WORLD HEALTH ORGANIZATION. Global Student Health Survey (GSHS). [S. l.: s. n.], 2013a. Disponível em: <https://www.cdc.gov/gshs/>. Acesso em: 22 de fevereiro de 2019.

WORLD HEALTH ORGANIZATION. Growth reference data for 5-19 years. 2013b. WHO. Disponível em: <https://www.who.int/growthref/en/>. Acesso em: 6 de setembro de 2019.

WORLD HEALTH ORGANIZATION. Obesity and overweight. 2018. Disponível em: <https://www.who.int/newsroom/fact-sheets/detail/obesity-and-overweight>. Acesso em: 10 de maio de 2019.

YANG, L; BOVET, P; MA, C; ZHAO, M; LIANG, Y; XI, B. Prevalence of underweight and overweight among young adolescents aged 12-15 years in 58 low-income and middle-income countries. Pediatric obesity, England, v. 14, n. 3, p. e12468, 2019. Disponível em: <https://doi.org/10.1111/ijpo.12468>. Acesso em: 27 de junho de 2019.

YEN, Yat; SHI, Yumin; SOEUNG, Bunly; SENG, Ratny; DY, Channy; SUY, Rathny; NGIN, Kimlong. The associated risk factors for underweight and overweight high school students in Cambodia. Diabetes \& metabolic syndrome, Netherlands, v. $12, \quad$ n. 5, p. 737-742, 2018. Disponível em: <https://doi.org/10.1016/j.dsx.2018.04.016>. Acesso em: 27 de junho de 2019.

ZAHEDI, Hoda; KELISHADI, Roya; HESHMAT, Ramin; MOTLAGH, Mohammad Esmaeil; RANJBAR, Shirin Hasani; ARDALAN, Gelayol; PAYAB, Moloud; CHINIAN, Mohammad; ASAYESH, Hamid; LARIJANI, Bagher; QORBANI, Mostafa. Association between junk food consumption and mental health in a national sample of Iranian children and adolescents: the CASPIAN-IV study. Nutrition (Burbank, Los Angeles County, Calif.), United States, v. 30, n. 11-12, p. 1391-1397, 2014. Disponível em: < https://doi.org/10.1016/j.nut.2014.04.014>. Acesso em: 27 de junho de 2019.

\section{(cc) EY}

Este trabalho está licenciado com uma Licença Creative Commons - Atribuição 4.0 Internacional. 